\title{
The redshift and afterglow of the extremely energetic gamma-ray burst GRB 080916C
}

J. Greiner ${ }^{1}$, C. Clemens ${ }^{1}$, T. Krühler ${ }^{1,2}$, A. von Kienlin ${ }^{1}$, A. Rau ${ }^{3}$, R. Sari ${ }^{4}$, D. B. Fox ${ }^{5}$, N. Kawai ${ }^{6}$, P. Afonso ${ }^{1}$, M. Ajello ${ }^{7}$, E. Berger ${ }^{8}$, S. B. Cenko ${ }^{9}$, A. Cucchiara ${ }^{5}$, R. Filgas $^{1}$, S. Klose ${ }^{10}$, A. Küpcü Yoldas ${ }^{11}$, G. G. Lichti ${ }^{1}$, S. Löw ${ }^{1}$, S. McBreen ${ }^{1,12}$, T. Nagayama ${ }^{13}$, A. Rossi ${ }^{10}$, S. Sato ${ }^{14}$, G. Szokoly ${ }^{1,15}$, A. Yoldaş ${ }^{1}$, and X.-L. Zhang ${ }^{1}$

1 Max-Planck-Institut für extraterrestrische Physik, Giessenbachstrasse 1, 85748 Garching, Germany e-mail: [jcg;cclemens;kruehler;azk; pafonso; filgas; grl; sloew;yoldas; zhangx]@mpe.mpg.de

2 Universe Cluster, Technische Universität München, Boltzmannstraße 2, 85748 Garching, Germany

3 Optical Observatories, California Inst. of Technology, 1200 E California Blvd, Pasadena, CA 91125, USA e-mail: arne@astro.caltech.edu

${ }^{4}$ Dept. of Theor. Astrophysics, California Inst. of Technology, 1200 East California Blvd., Pasadena, CA 91125, USA e-mail: sari@tapir.caltech.edu

5 Department of Astronomy \& Astrophysics, Pennsylvania State University, 525 Davey Lab, University Park, PA 16802, USA e-mail: dfox@astro.psu.edu

6 Dept. of Physics, Tokyo Inst. of Technology, 2-12-1 Ookayama, Meguro-ku, Tokyo 152-8551, Japan e-mail: nkawai@phys.titech.ac.jp

7 SLAC/KIPAC, 2575 Sand Hill Road, Menlo Park, CA 94025, USA e-mail: majello@slac.stanford.edu

8 Harvard University, 60 Garden Street, Cambridge, MA 02138, USA e-mail: eberger@cfa.harvard.edu

9 Department of Astronomy, University of California, Berkeley, CA 94720, USA e-mail: cenko@astro.berkeley.edu

10 Thüringer Landessternwarte Tautenburg, Sternwarte 5, 07778 Tautenburg, Germany e-mail: [klose;rossi]@tls-tautenburg.de

11 ESO, Karl-Schwarzschild-Str. 2, 85740 Garching, Germany e-mail: ayoldas@eso.org

12 School of Physics, University College Dublin, Belfield, Dublin 4, Ireland e-mail: sheila.mcbreen@ucd.ie

13 Dept. of Astronomy, Kyoto University, Sakyo-ku, Kyoto 606-8502, Japan e-mail: nagayama@kusastro.kyoto-u.ac.jp

14 Department of Astrophysics, Nagoya University, Furo-cho, Chikusa-ku, Nagoya 464-8602, Japan e-mail: ssato@z.phys.nagoya-u.ac.jp

15 Eötvös Univ., 1117 Budapest, Pazmany P. stny. 1/A, Hungary e-mail: szgyula@elte.hu

Received 22 December 2008 / Accepted 7 February 2009

ABSTRACT

Context. The detection of GeV photons from gamma-ray bursts (GRBs) has important consequences for the interpretation and modelling of these most-energetic cosmological explosions. The full exploitation of the high-energy measurements relies, however, on accurate knowledge of the distance to the events.

Aims. Here we report on the discovery of the afterglow and subsequent redshift determination of GRB 080916C, the first GRB detected by the Fermi Gamma-Ray Space Telescope with high significance detection of photons at energies $>0.1 \mathrm{GeV}$.

Methods. Observations were done with the 7-channel "Gamma-Ray Optical and Near-infrared Detector" (GROND) at the $2.2 \mathrm{~m}$ MPI/ESO telescope, the SIRIUS instrument at the Nagoya-SAAO $1.4 \mathrm{~m}$ telescope in South Africa, and the GMOS instrument at Gemini-S.

Results. The afterglow photometric redshift of $z=4.35 \pm 0.15$, based on simultaneous 7-filter observations with GROND, places GRB 080916C among the top 5\% most distant GRBs and makes it the most energetic GRB known to date. The detection of GeV photons from such a distant event is unexpected because of the predicted opacity due to interaction with the extragalactic background light. The observed gamma-ray variability in the prompt emission, together with the redshift, suggests a lower limit for the Lorentz factor of the ultra-relativistic ejecta of $\Gamma>1090$. This value rivals any previous measurements of $\Gamma$ in GRBs and strengthens the extreme nature of GRB 080916C.

Key words. techniques: photometric - gamma rays: bursts

\section{Introduction}

Long-duration gamma-ray bursts (GRBs) are the high-energy signatures of the death of some massive stars, and they emit the bulk of their radiation in the $300-800 \mathrm{keV}$ band. In a few events, emission up to $\sim 100 \mathrm{MeV}$ has been detected, e.g., with SMM (Harris \& Share 1998), COMPTEL (Hoover et al. 2005), EGRET (Kaneko et al. 2008), and recently with AGILE (Giuliani et al. 2008). These high-energy photons offer unique 


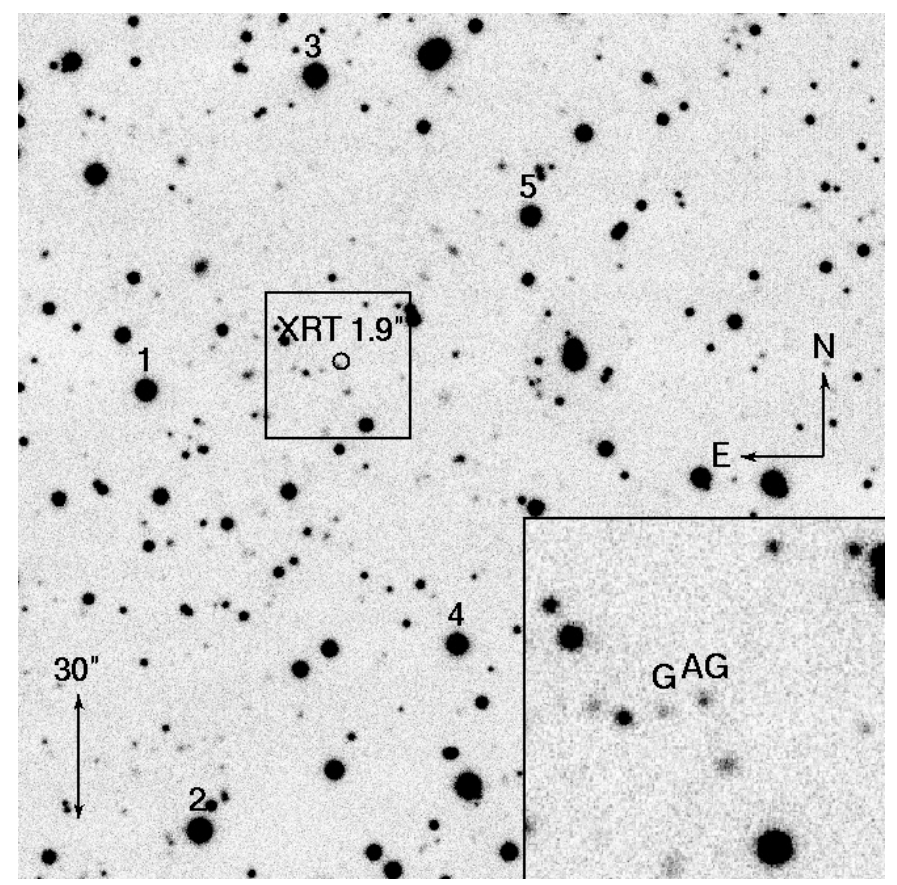

Fig. 1. $i^{\prime}$-band image of the afterglow of GRB 080916C obtained with the 7-channel imager GROND at the $2.2 \mathrm{~m}$ telescope on La Silla / Chile $32 \mathrm{~h}$ after the burst. The circle denotes the $S$ wift/XRT error box. The local standard stars of Table 2 are labeled. A zoom into the innermost region is shown in the bottom-right, with the afterglow (AG) and a galaxy (G) 4" from the afterglow labeled.

access to the physics of GRBs. Firstly, the shape of the spectrum provides direct information about the gamma-ray emission mechanism (Pe'er et al. 2007; Giannios 2008). Secondly, highenergy photons can place tight constraints on the Lorentz factor of the ejecta via the pair-production threshold. Furthermore, in some cases, the origin of the high-energy component differs from that of the low-energy emission (e.g., GRB 941017; Gonzalez et al. 2003) or the high-energy photons arrive with a significant time delay (e.g., >1 h in GRB 940217; Hurley et al. 1999). The formation of these properties is far from understood and can only be addressed with an increasing number of bursts with $\mathrm{GeV}$ detections. Finally, the search for signatures of absorption against the intergalactic UV background light using the shape of the high-energy spectrum, as well as the search for quantum gravity dispersion effects over cosmic distances in the light curve imply much broader scientific interest (Abdo et al. 2009).

An important prerequisite to any interpretation of the $\mathrm{GeV}$ component of a burst is accurate knowledge of the distance. Only few of the previously detected GRBs with high-energy emission had identified optical afterglows, as the localization capabilities of high-energy missions were insufficient to facilitate rapid follow-up observations.

The recently launched Fermi Gamma-Ray Space Telescope has the ability to localise high-energy events using the Large Area Telescope (LAT) and to measure spectra over a wide energy range in combination with the Gamma-Ray Monitor (GBM) ( $8 \mathrm{keV}$ to $300 \mathrm{GeV}$ ). The Swift satellite (Gehrels et al. 2004) can also slew rapidly to LAT locations and provide positions with arcsec-accuracy by the detection of the X-ray afterglow, facilitating and dramatically enhancing the likelihood of a distance measurement.

The bright GRB $080916 \mathrm{C}$ was detected by the GBM on 2008 Sep. 16th, at 00:12:45 UT (Goldstein \& van der Horst 2008). The

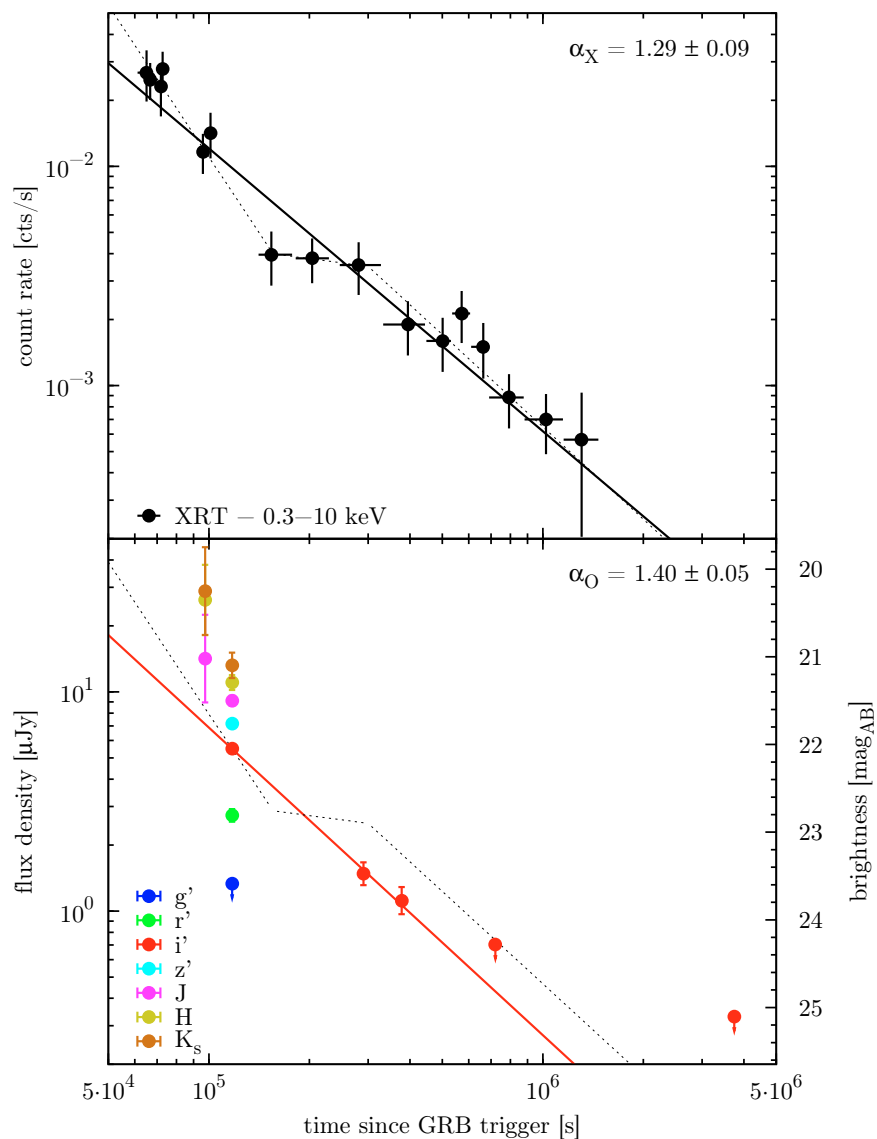

Fig. 2. X-ray (upper panel; Evans et al. 2007) and optical/NIR light curve (lower panel) of the GRB 080916C afterglow. The solid lines mark the best fit power laws to the X-ray and $i^{\prime}$-band data (labeled in the upper right corner). The power law segments as given by Swift (Stratta et al. 2008) are shown as dashed lines; they were scaled to the $i^{\prime}$-band in the lower panel to show that this 3-segment power law does not fit the optical data, i.e. the apparent X-ray plateau phase is not detected in the optical data. However, the X-ray light curve can also be fit with a single power law, with only marginally larger $\chi_{\text {red }}^{2}$, as compared to the 3 -segment powerlaw, which results in $\alpha_{\mathrm{X}}=1.29 \pm 0.09$. The optical decay is mainly constrained by the three $i^{\prime}$-band data points. The $J H K_{\mathrm{S}}$ observations of SIRIUS and GROND during the first night are consistent with this decay.

burst was located in in the field of view of the LAT and emission above $100 \mathrm{MeV}$ was quickly localised (Tajima et al. 2008). Follow-up observations with the Swift X-ray telescope (XRT) provided an X-ray afterglow candidate (Kennea 2008) which subsequently led to the discovery of a faint optical/NIR source with GROND (Clemens et al. 2008a) and SIRIUS (Nagayama 2008). Further monitoring in both X-rays (Stratta et al. 2008) and in the optical (Clemens et al. 2008b) established the fading and confirmed the source to be the afterglow of GRB 080916C.

GRB 080916C was also detected by other satellites in addition to Fermi (Hurley et al. 2008): AGILE (MCAL, SuperAGILE, and ACS), RHESSI, INTEGRAL (SPI-ACS), Konus-Wind, and MESSENGER. The preliminary analysis of the GBM integrated spectrum over a duration $\left(T_{90}\right)$ of $66 \mathrm{~s}$ results in a best fit Band function (Band et al. 1993) with $E_{\text {peak }}=$ $424 \pm 24 \mathrm{keV}$, a low-energy photon index $\alpha=-0.91 \pm 0.02$ and a high-energy index $\beta=-2.08 \pm 0.06$, giving a fluence of $1.9 \times 10^{-4} \mathrm{erg} \mathrm{cm}^{-2}$ in the $8 \mathrm{keV}-30 \mathrm{MeV}$ range (van der Horst $\&$ Goldstein 2008). The spectral results reported by RHESSI and Konus-Wind (Golenetskii et al. 2008) are in broad agreement. 
Table 1. Log of the observations.

\begin{tabular}{llccc}
\hline \hline $\begin{array}{l}\text { Date/Time } \\
\text { (UT in 2008) }\end{array}$ & Telescope/Instrument & Filter & $\begin{array}{c}\text { Exposure } \\
(\mathrm{min})\end{array}$ & \multicolumn{1}{c}{$\begin{array}{c}\text { Brightness } \\
(\mathrm{mag})^{(a)}\end{array}$} \\
\hline Sep. 17 02:53-03:43 & IRSF/SIRIUS & $J H K_{\mathrm{S}}$ & 50.0 & $21.0 \pm 0.5 / 20.4 \pm 0.4 / 20.3 \pm 0.5$ \\
Sep. 17 07:57-09:39 & MPI/ESO 2.2 m/GROND & $g^{\prime} r^{\prime} i^{\prime} z^{\prime}$ & 75.0 & $>23.6 / 22.81 \pm 0.07 / 22.05 \pm 0.05 / 21.76 \pm 0.05$ \\
Sep. 17 07:57-09:39 & MPI/ESO 2.2 m/GROND & $J H K_{\mathrm{S}}$ & 60.0 & $21.50 \pm 0.06 / 21.29 \pm 0.08 / 21.10 \pm 0.15$ \\
Sep. 19 08:04-09:42 & MPI/ESO 2.2 m/GROND & $g^{\prime} r^{\prime} i^{\prime} z^{\prime}$ & 79.4 & $>23.6 />23.8 / 23.47 \pm 0.13 />23.8$ \\
Sep. 19 08:04-09:42 & MPI/ESO 2.2 m/GROND & $J H H K_{S}$ & 64.0 & $>21.9 />21.2 />20.5$ \\
Sep. 20 08:42-09:42 & MPI/ESO 2.2 m/GROND & $g^{\prime} r^{\prime} i^{\prime} z^{\prime}$ & 50.0 & $>23.9 />24.2 / 23.78 \pm 0.16 />23.8$ \\
Sep. 20 08:42-09:42 & MPI/ESO 2.2 m/GROND & $J H K_{\mathrm{S}}$ & 40.0 & $>22.5 />21.5 />20.6$ \\
Sep. 24 07:32-09:31 & MPI/ESO 2.2 m/GROND & $g^{\prime} r^{\prime} i^{\prime} z^{\prime}$ & 100.1 & $>25.0 />24.5 />24.3 />23.9$ \\
Sep. 24 07:32-09:31 & MPI/ESO 2.2 m/GROND & $J H K_{\mathrm{S}}$ & 74.2 & $>22.2 />21.5 />20.8$ \\
Oct. 29 07:59-08:31 & Gemini-S/GMOS & $i^{\prime}$ & 24.0 & $>25.1$ \\
\hline
\end{tabular}

${ }^{(a)}$ Not corrected for Galactic foreground reddening of $E(B-V)=0.32 \mathrm{mag}$ (Schlegel et al. 1998). All magnitudes are given in the AB system.

Table 2. Local photometric standards within $2^{\prime}$ of the GRB.

\begin{tabular}{ccccccccc}
\hline \hline No & Coordinates (J2000) & $g^{\prime}$ & $r^{\prime}$ & $i^{\prime}$ & $z^{\prime}$ & $J$ & $H$ & $K_{\mathrm{S}}$ \\
\hline 1 & $07: 59: 28.97-56: 38: 24.0$ & $17.59 \pm 0.01$ & $16.82 \pm 0.01$ & $16.50 \pm 0.01$ & $16.27 \pm 0.01$ & $16.24 \pm 0.01$ & $15.98 \pm 0.01$ & $16.36 \pm 0.01$ \\
2 & $07: 59: 27.40-56: 40: 10.1$ & $17.18 \pm 0.01$ & $16.18 \pm 0.01$ & $15.79 \pm 0.01$ & $15.52 \pm 0.01$ & $15.37 \pm 0.01$ & $15.00 \pm 0.01$ & $15.38 \pm 0.01$ \\
3 & $07: 59: 24.01-56: 37: 08.0$ & $17.11 \pm 0.01$ & $16.31 \pm 0.01$ & $15.98 \pm 0.01$ & $15.72 \pm 0.01$ & $15.67 \pm 0.01$ & $15.40 \pm 0.01$ & $15.77 \pm 0.01$ \\
4 & $07: 59: 19.84-56: 39: 25.3$ & $17.90 \pm 0.01$ & $16.92 \pm 0.01$ & $16.54 \pm 0.01$ & $16.28 \pm 0.01$ & $16.15 \pm 0.01$ & $15.84 \pm 0.01$ & $16.20 \pm 0.01$ \\
5 & $07: 59: 17.70-56: 37: 41.9$ & $18.11 \pm 0.01$ & $17.09 \pm 0.01$ & $16.71 \pm 0.01$ & $16.45 \pm 0.01$ & $16.36 \pm 0.01$ & $16.02 \pm 0.01$ & $16.44 \pm 0.01$ \\
\hline
\end{tabular}

The measurements of the high-energy emission from GRB 080916C by the instruments of the Fermi Gamma-Ray Space telescope are described in Abdo et al. (2009). Here we report on the discovery of the optical/NIR afterglow of GRB 080916C, the measurement of its redshift, and consequently on the recognition of its extreme explosion energy and the large Lorentz factor of its relativistic outflow.

\section{The GRB afterglow}

The first ground-based imaging was obtained with the Simultaneous 3-colour ( $J H K$ ) InfraRed Imager for Unbiased Survey (SIRIUS, Nagayama et al. 2003) on the Nagoya-SAAO $1.4 \mathrm{~m}$ telescope (IRSF). GROND, a simultaneous 7-channel imager (Greiner et al. 2009) mounted at the $2.2 \mathrm{~m} \mathrm{MPI/ESO}$ telescope at La Silla (Chile), started observing about $30.75 \mathrm{~h}$ after the GRB. The imaging sequence consisted of a series of sixteen $375 \mathrm{~s}$ integrations in the $g^{\prime} r^{\prime} i^{\prime} z^{\prime}$ channels with gaps of about $45 \mathrm{~s}$. In parallel, the $J H K_{\mathrm{S}}$ channels were operated with $10 \mathrm{~s}$ integrations, separated by $5 \mathrm{~s}$. Late-time imaging was obtained with the Gemini-South telescope + Gemini Multi-Object Spectrograph (GMOS-South) on 29 Oct. 2008, taking eight 180 s exposures. Data reduction was done using IRAF routines. Photometric calibration of the GROND $g^{\prime}, r^{\prime}, i^{\prime}, z^{\prime}$ bands was performed using the spectrophotometric standard stars SA100-241 and SA97249, while that of $J H K_{S}$ was done against 2MASS (Table 2). The magnitudes of the selected 2MASS stars were transformed into the GROND filter system and finally into $A B$ magnitudes using $J(A B)=J($ Vega $)+0.91, H(A B)=H($ Vega $)+1.38$, $K(A B)=K($ Vega $)+1.81$ (Greiner et al. 2009). Systematic errors are $\pm 0.02 \mathrm{mag}$ for $g^{\prime} r^{\prime} i^{\prime} z^{\prime}$, and $\pm 0.05 \mathrm{mag}$ for $J H K_{\mathrm{s}}$.

A comparison of GROND observations from Sep. 17 and 19, 2008 clearly reveals a fading source inside the $S$ wift/XRT error box (Fig. 1), with coordinates RA $(\mathrm{J} 2000.0)=07^{\mathrm{h}} 59^{\mathrm{m}} 23^{\mathrm{s}} .32$, Dec $(\mathrm{J} 2000.0)=-56^{\circ} 38^{\prime} 18^{\prime \prime} .0$ ( $0{ }^{\prime} .5$ error $)$. The decay between 1.3 to $4 \mathrm{~d}$ after the GRB is well described by a single power law with
Table 3. Results of the spectral energy distribution fitting without dust and with dust models of various types.

\begin{tabular}{lcccc}
\hline \hline Dust model & Redshift & $\beta^{1}$ & $\begin{array}{c}A_{V}^{\text {host }} \\
(\mathrm{mag})\end{array}$ & $\chi_{\text {red }}^{2}$ \\
\hline none & $4.35_{-0.13}^{+0.12}$ & $0.38_{-0.19}^{+0.20}$ & - & 1.04 \\
Milky Way & $4.35_{-0.16}^{+0.12}$ & $0.38_{-0.23}^{+0.21}$ & $0.0_{-0.0}^{+0.4}$ & 1.04 \\
Large Magellanic Cloud & $4.28_{-0.15}^{+0.15}$ & $0.34_{-0.24}^{+0.19}$ & $0.1_{-0.1}^{+0.4}$ & 0.95 \\
Small Magellanic Cloud & $4.35_{-0.26}^{+0.13}$ & $0.38_{-0.28}^{+0.13}$ & $0.0_{-0.0}^{+0.2}$ & 1.04 \\
\hline
\end{tabular}

${ }^{1}$ Errors are at the $2 \sigma$ confidence level.

$\alpha_{O}=1.40 \pm 0.05$ (Fig. 2), compatible within the errors to the X-ray decay slope $\alpha_{\mathrm{X}}=1.29 \pm 0.09$.

A spectral energy distribution (SED) was constructed using the GROND magnitudes from the first night of observations (Table 1). The photometrically calibrated data (Table 2) were corrected for the Galactic foreground reddening of $E(B-V)=$ 0.32 mag (Schlegel et al. 1998) corresponding to an extinction of $A_{V}=0.98 \mathrm{mag}$ and fit by an intrinsic power law $\left(F_{v} \propto v^{-\beta}\right)$ plus three different dust models, as well as without extinction (Table 3). The $i^{\prime}$ to $K_{\mathrm{S}}$ band data are best fit with a power law slope of $\beta=0.38 \pm 0.20$ and no host-intrinsic extinction. The $i^{\prime}$ to $r^{\prime}$ band measurements deviate significantly and can be best explained with a Ly- $\alpha$ break at $z=4.35 \pm 0.15$ (see Table 3 and Fig. 3, and the rejected alternative explanations given in the figure caption). The redshift values resulting from all the fitted models are compatible, and the redshift error includes already the dependence on the error of the photon index as well as foreground-extinction correction (Fig. 4). The $g^{\prime}$-band upper limit is consistent with the high-redshift result, though it is not deep enough to constrain the fit.

As an alternative interpretation, the $r^{\prime}$-band drop could be attributed to reddening in the host galaxy, caused either by substantial UV absorption or a strong broad absorption feature like 


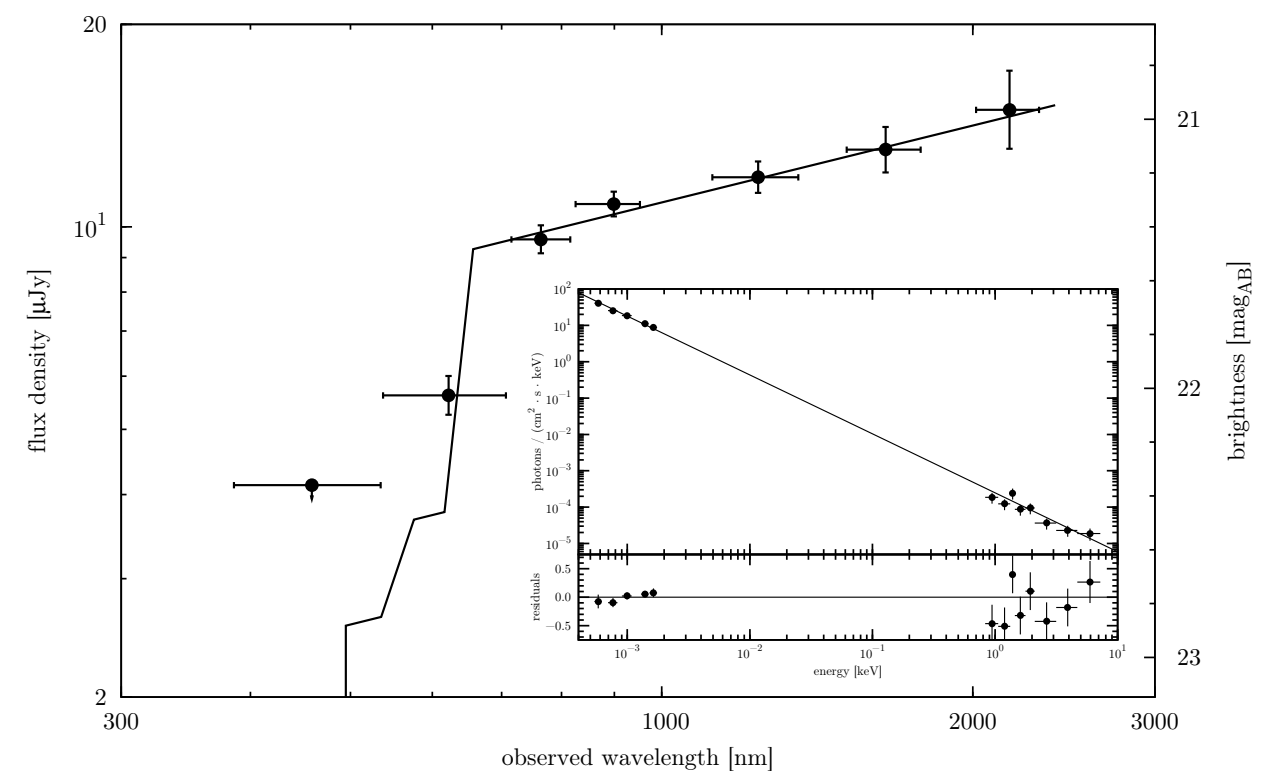

Fig. 3. Spectral energy distribution of the afterglow, derived from the coaddition of all GROND exposures from the first night (Sep. 17, 2008). The SED is best fit with a power law of spectral index $\beta=0.38$, no extinction, and Ly $\alpha$ absorption at a redshift of $z=4.35 \pm 0.15$. The inset shows that the best-fit GROND power law connects without break or offset to the Swift/XRT data, supporting the correct modelling of the GROND SED and the re-interpretation of the X-ray spectrum.

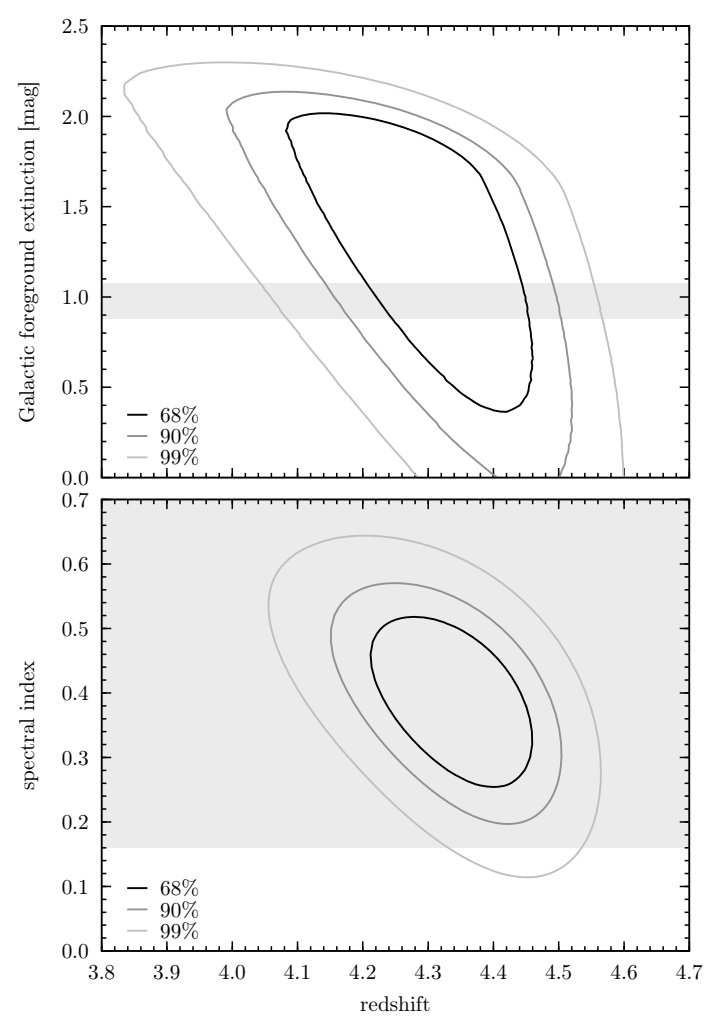

Fig. 4. Contour plot of the foreground $A_{\mathrm{V}}$ (upper panel) and spectral index (lower panel) from the spectral energy distribution fit against the redshift. This shows that the photo- $z$ determination is stable against uncertainties in the correction for the galactic foreground extinction, as well as the powerlaw index. The $68 \%, 90 \%$ and $99 \%$ confidence contours are plotted. The shaded area in the upper panel shows the $A_{\mathrm{V}}$ range according to (Schlegel et al. 1998). In the bottom panel the shaded area shows the power-law spectral index from the X-ray spectral fits.

that at $2175 \AA$ (Krühler et al. 2008). However, the resulting host extinction corrected spectral slope of $\beta \lesssim 0$.0 would be incompatible with most theoretical models (Sari et al. 1998) and with the X-ray spectrum. The lack of curvature in the $i^{\prime}-K_{\mathrm{S}} \mathrm{SED}$, and its extrapolation to the X-ray data, provide additional arguments against host extinction. Similarly, a spectral break cannot easily explain the $r^{\prime}-i^{\prime}$ colour without redshift as the difference in power law index would be 2.5 , much larger than predicted by theory (Sari et al. 1998). In addition, the steep power law would significantly underpredict the observed X-ray fluxes. The inset of Fig. 3 shows that the best-fit GROND power law connects without break or offset to the Swift/XRT data, supporting the correct modelling of the GROND SED.

No X-ray measurements of the afterglow are available during the time of the initial GROND epoch. However, we can use the SIRIUS $J H K_{\mathrm{S}}$-band brightness as well as a back-extrapolation of the afterglow decay slope to re-scale the GROND SED to the earlier time when XRT measurements are available. The Swift/XRT spectrum from 61-102 ks post-burst has been reported to be well fit with an absorbed power law spectrum with photon index $\Gamma_{\mathrm{X}}=2.1_{-0.7}^{+0.9}$ and a column density of $N_{\mathrm{H}}=3.7_{-1.1}^{+3.3} \times$ $10^{21} \mathrm{~cm}^{-2}$ (Stratta et al. 2008). Using the result of no excess extinction, we re-fit the X-ray spectrum with the column density fixed to the galactic foreground value $\left(N_{\mathrm{H}}=1.5 \times 10^{21} \mathrm{~cm}^{-2}\right)$, and obtain $\Gamma_{\mathrm{X}}=1.49_{-0.34}^{+0.31}$, consistent with the slope of the GROND SED (note that the spectral index $\beta$ is related to the photon index $\Gamma$ from the X-ray spectral fitting by $\left.\beta_{X}=\Gamma_{X}-1\right)$. The GROND and XRT combined SED is compatible with a single power law over the complete spectral range (see inset of Fig. 3).

No counterpart or host galaxy was detected seven weeks after the burst at the position of the optical/NIR afterglow, with a two-sigma upper limit of $i^{\prime}>25.1 \mathrm{mag}$ within a $1.1^{\prime \prime}$-radius aperture centred at the afterglow position. This is not surprising given the brightness distribution of the known GRB host galaxies (Savaglio et al. 2009; the brightness limit places a loose lower limit on the redshift of $z>1$.

We note that the nearest object visible on our images is a galaxy at 4 " distance to the East. We obtained an optical spectrum of this galaxy beginning at 05:00 UT on 7 November 2008, using the Gemini-South telescope +GMOS-S. We obtained two spectra of $900 \mathrm{~s}$ each with the R400 grating centred at $8000 \AA$, 


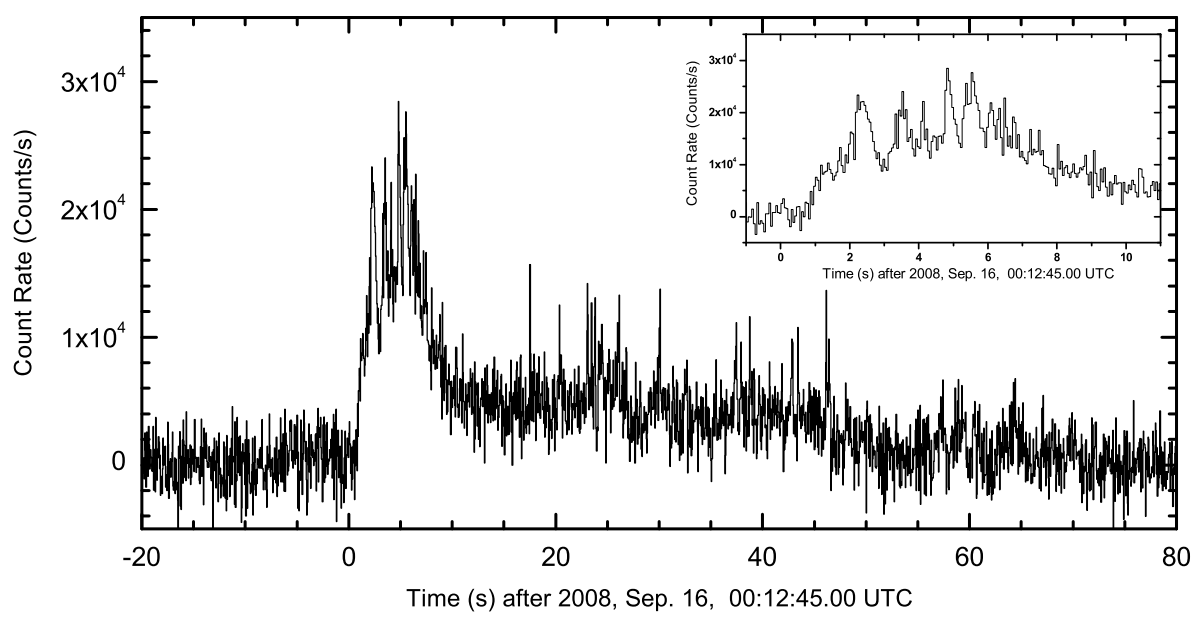

Fig. 5. Prompt emission light curve in the $80 \mathrm{keV}-30 \mathrm{MeV}$ energy band as measured with INTEGRAL SPI-ACS at $50 \mathrm{~ms}$ resolution. The inset shows a zoom of the main peak. Variability on time scale as short as $100 \mathrm{~ms}$ is visible - we measure several $6 \sigma$ flux variations relative to the neighbouring data bins on the $100 \mathrm{~ms}$ time scale. a second-order blocking filter in place, and with the slit oriented to provide simultaneous coverage of the afterglow position. Observations were carried out at relatively high airmass, with 1.7" seeing and variable sky background, and consist of two spectra of $900 \mathrm{~s}$ each. Bias subtraction, flat-fielding, and wavelength calibration were performed using the GMOS reduction package under the IRAF environment. The wavelength solution was derived using a CuAr lamp spectrum taken immediately after the science spectra. Following extraction, the two onedimensional spectra were coadded to increase signal-to-noise, achieving a median $S / N$ of 15.8 over the 6000-10000 $\AA$ wavelength range. We clearly detect the continuum of the resolved galaxy at wavelengths greater than $6100 \AA$, placing an upper limit on the redshift of this galaxy of $z<4.0$, based on the absence of Lyman-alpha absorption. This is below the $99 \%$ confidence range for the redshift of GRB 080916C (Fig. 4), and thus this galaxy is not related to this burst.

\section{Discussion and conclusions}

It is widely believed that long GRBs are produced in the gravitational collapse of a massive star into a neutron star or black hole. It has been argued in the past that the observed total energy in GRBs requires that the emission is relativistic. GRB 080916C is unrivaled by all previous events in this respect: with its observed fluence of $1.9 \times 10^{-4} \mathrm{erg} \mathrm{cm}^{-2}$ in the $8 \mathrm{keV}-30 \mathrm{MeV}$ band (van der Horst \& Goldstein 2008), and redshift of $4.35 \pm 0.15$, its total isotropic energy release is a staggering $6.5 \times 10^{54} \mathrm{erg}$, corresponding to $4 M_{\odot} \times \mathrm{c}^{2}$ !

The afterglow decay slope is unlikely a post-jet break decay, which allows one to assume that the jet break occurs after the final XRT observations at $2 \times 10^{6} \mathrm{~s}$. A break after this time would place a lower limit on the jet half-opening angle of $6.1 \pm 0.1$ (ISM surrounding with density of $1 \mathrm{~cm}^{-3}$ ) or $2.2 \pm 0.1$ (wind medium), which in turn implies lower limits for the beaming-corrected energy release of $(3.7 \pm 0.1) \times 10^{52} \mathrm{erg}$ $(\mathrm{ISM})$ or $(4.9 \pm 0.1) \times 10^{51} \mathrm{erg}$ (wind). While we cannot distinguish between these two cases, we note that the energy for the ISM case is extremely high, exceeding the previous record holders GRB 080721 ( $1 \times 10^{52} \mathrm{erg}$, Starling et al. 2009), 990123 $\left(6 \times 10^{51} \mathrm{erg}\right.$, Kulkarni et al. 1999) and $050904\left(7 \times 10^{51} \mathrm{erg}\right.$, Tagliaferri et al. 2005) by several factors. For the wind medium case, a jet break is expected to be very smooth, and might have started near the end of our coverage; thus the real opening angle may not differ much from our lower limit. Using the $\alpha-\beta$ relations it is most likely that we are in the pre-jet break phase when the first GROND data were taken. Even though the error bars of $\beta$ are large, the data are best compatible with a wind model and the cooling frequency above the X-ray band.

If the emission were non-relativistic, the required photon field at the burst location would be optically thick to pairproduction ("compactness problem"; Ruderman 1975). It has been recognised that in addition to the annihilation of photons into electron/position pairs, the scattering of photons by either the electron or the positron created in the annihilation process, contributes to the optical depth of high-energy photons (Lithwick \& Sari 2001). In fact, this latter limit is in many cases more constraining than the pure annihilation limit.

The most sensitive instrument that detected high-energy photons from GRB 080916C was the anti-coincidence system (ACS) of the spectrometer onboard INTEGRAL (SPI) (Rau et al. 2005). At its native time resolution SPI-ACS recorded at peak more than 1200 counts per $50 \mathrm{~ms}$ in the $80 \mathrm{keV}-30 \mathrm{MeV}$ energy range. This allowed the detection of variability on time scales as short as $100 \mathrm{~ms}$ with high statistical significance (see inset of Fig. 5). Using Eq. (9) of Lithwick \& Sari (2001) and the photon index of $\beta=-2.08$ as measured from GBM (van der Horst \& Goldstein 2008), we estimate a lower limit on the Lorentz factor of the ejecta of $\Gamma>1090$. The previously highest limit on the Lorentz factor of a GRB with measured (rather than adopted) redshift using this method has been $\Gamma>410$ (Lithwick \& Sari 2001) for GRB 971214 at $z=3.42$, for which the additional assumption had to be made that the photon spectrum actually extended to very high energies.

Over the last two years, an alternative method to determine the initial Lorentz factor has been employed. This method is based on observations of the rising part of optical afterglows to determine when the blast wave has decelerated; the corresponding Lorentz factor at the time of the deceleration is expected to be half of the initial Lorentz factor $\Gamma_{0}$ (Sari \& Piran 1999). Assuming an ISM type environment, this method provided $\Gamma_{0} \approx 400$ for GRB 060418 and 060607 (Molinari et al. 2007), $\Gamma_{0}=160$ for GRB 070802 (Krühler et al. 2008), $\Gamma_{0}=120$ for GRB 080129 (Greiner et al. 2009), $\Gamma_{0}=200$ for GRB 071031 (Krühler et al. 2009) and $\Gamma_{0}=230-370$ for GRB 060605 (Ferrero et al. 2009). Yet another method is based on the evolution of the thermal emission component in the prompt emission of GRBs (Pe'er et al. 2007), and also provides similarly low values of $\Gamma$. It is interesting to note that our lower limit on $\Gamma$ for GRB 080916C is substantially higher than values determined by other methods. Whether or not this is related to the $\mathrm{GeV}$ emission in GRB 080916C remains to be seen. 
The high signal-to-noise ratio of the SPI-ACS data is also ideal for estimating the variability of the light curve, a quantity that has been shown to correlate with the isotropic equivalent

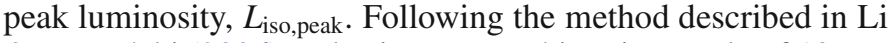
\& Paczyński (2006) and using a smoothing time scale of 13.7 s, we derived a variability index of $V=-2.26$ and a resulting $L_{\text {iso,peak }}=(1.23 \pm 0.32) \times 10^{52} \mathrm{erg} \mathrm{s}^{-1}(80 \mathrm{keV}-30 \mathrm{MeV})$. Using the observed $256 \mathrm{~ms}$ peak flux from Konus (Golenetskii et al. 2008), we derive $L_{\text {iso,peak }}=2 \times 10^{53} \mathrm{erg} \mathrm{s}^{-1}(20 \mathrm{keV}-10 \mathrm{MeV})$.

For the future, the synergy of the detection of GRBs with $\mathrm{GeV}$ emission coupled with the ability to localise and determine redshifts for these events will be extremely interesting as both $\Gamma$ determination methods can be applied, providing a consistency check of our picture of the GRB and afterglow emission process.

Acknowledgements. T.K. acknowledges support by the DFG cluster of excellence "Origin and Structure of the Universe". Part of the funding for GROND (both hardware as well as personnel) was generously granted from the LeibnizPrize to Prof. G. Hasinger (DFG grant HA 1850/28-1). Av.K. acknowledges funding through DLR 50 QV 0301, and X.L.Z. through DLR 50 OG 0502. S.M.B. acknowledges an EU Marie Curie Fellowship in Framework 6. This work made use of data supplied by the UK Swift Science Data Centre at the University of Leicester. We thank X. Wu for a discussion oon the $\alpha-\beta$ relation.

Facilities: Max Planck: $2.2 \mathrm{~m}$ (GROND), Gemini: South (GMOS), SAAO: Nagoya (SIRIUS), INTEGRAL (SPI-ACS).

\section{References}

Abdo, A. A., Ackermann, M., Ajello, M., et al. 2009, Science, in press Band, D., Matteson, J., Ford, L., et al. 1993, ApJ, 413, 281

Clemens, C., Rossi, A., Greiner, J., et al. 2008a, GCN, 8257

Clemens, C., Rossi, A., Greiner, J., et al. 2008b, GCN, 8272
Evans, P. A., Beardmore, A. P., Page, K. L., et al. 2007, A\&A, 469, 379 Ferrero, P., Klose, S., Kann, D. A., et al. 2009, A\&A, in press

Gehrels, N., Chincarini, G., Giommi, P., et al. 2004, ApJ, 621, 558 Giannios, D. 2008, A\&A, 488, L55

Giuliani, A., Mereghetti, S., Fornari, F., et al. 2008, A\&A, 491, L25 Goldstein, A., \& van der Horst, A. 2008, GCN, 8245

Golenetskii, S., Aptekar, R., Mazets, E., et al. 2008, GCN, 8258

Gonzalez, M. M., Dingus, B. L., Kaneko, Y., et al. 2003, Nature, 424, 749

Greiner, J., Bornemann, W., Clemens, C., et al. 2008, PASP, 120, 405

Greiner, J., Krühler, T., McBreen, S., et al. 2009, ApJ, 693, 1912

Harris, M. J., \& Share, G. H. 1998, ApJ, 494, 724

Hoover, A. S., Kippen, R. M., \& McConnell, M. L. 2005, Il Nuovo Cimento C, 28,825

Hurley, K., Dingus, B. L., Mukherjee, R., et al. 1994, Nature, 372, 652

Hurley, K., Goldsten, J., Golenetskii, S., et al. 2008, GCN, 8251

Kaneko, Y., Gonzalez, M. M., Preece, R. D., et al. 2008, ApJ, 677, 1168

Kennea, J. A. 2008, GCN, 8253

Krühler, T., Küpcü Yoldaş, A., Greiner, J., et al. 2008, ApJ, 685, 376

Krühler, T., Greiner, J., McBreen, S., et al. 2009, ApJ, in press [arXiv: 0903.1184]

Kulkarni, S. R., Djorgovski, S. G., Odewahn, S. C., et al. 1999, Nature, 398, 389 Li, L.-X., \& Paczyński, B. 2006, MNRAS, 366, 219

Lithwick, Y., \& Sari, R. 2001, ApJ, 555, 540

Molinari, E., Vergani, S. D., Malesani, D., et al. 2007, A\&A, 469, L13

Nagayama, T., Nagashima, C., Nakajima, Y., et al. 2003, Proc. SPIE, 4841, 459

Nagayama, T. 2008, GCN, 8274

Pe'er, A., Ryde, F., Wijers, R. A. M. J., et al. 2007, ApJ, 664, L1

Rau, A., Kienlin, A. V., Hurley, K., \& Lichti, G. G. 2005, A\&A 438, 1175

Ruderman, M. 1975, Ann. NY Acad. Sci., 262, 164

Sari, R., \& Piran, T. 1999, ApJ, 520, 641

Sari, R., Piran, T., \& Narayan, R. 1998, ApJ, 497, L17

Savaglio, S., Glazebrook, K., \& Le Borgne, D. 2009, ApJ, 691, 182

Schlegel, D., Finkbeiner, D., \& Davis, M. 1998, ApJ, 500, 525

Starling, R. L. C., Rol, E., van der Horst, A. J., et al. 2009, MNRAS, submitted, [arXiv: 0812.2490]

Stratta, G., Perri, M., Preger, B., et al. 2008, GCN Report, 166.1

Tagliaferri, G., Antonelli, L. A., Chincarini, G., et al. 2005, A\&A, 443, L1

Tajima, H., Bregeon, J., Chiang, J., et al. 2008, GCN, 8246

van der Horst, A., \& Goldstein, A. 2008, GCN, 8278 